\title{
Prescribing Associated with High-Risk Opioid Exposures Among Non-cancer Chronic Users of Opioid Analgesics: a Social Network Analysis
}

\author{
Keiki Hinami, MD, MS ${ }^{1,2,3}$, Michael J. Ray, MPH ${ }^{1,2}$, Kruti Doshi, MBA ${ }^{1,2}$, Maria Torres, MD4, \\ Steven Aks, DO ${ }^{5}$, John J. Shannon, $M D^{7}$, and William E. Trick, MD ${ }^{1,2}$ \\ 'Department of Medicine, Cook County Health, Chicago, IL, USA; ${ }^{2}$ Collaborative Research Unit, Cook County Health , Chicago, IL, USA; \\ ${ }^{3}$ Section of Preventive Medicine, Cook County Health, Chicago, IL, USA; ${ }^{4}$ Department of Anesthesiology, Division of Pain Management, Cook \\ County Health , Chicago, IL, USA; ${ }^{5}$ Department of Emergency Medicine, Division of Medical Toxicology, Cook County Health , Chicago, IL, USA.
}

BACKGROUND: The continued rise in fatalities from opioid analgesics despite a steady decline in the number of individual prescriptions directing $\geq 90$ morphine milligram equivalents (MME)/day may be explained by patient exposures to redundant prescriptions from multiple prescribers.

OBJECTIVES: We evaluated prescribers' specialty and social network characteristics associated with high-risk opioid exposures resulting from single-prescriber high-daily dose prescriptions or multi-prescriber discoordination.

DESIGN: Retrospective cohort study.

PARTICIPANTS: A cohort of prescribers with opioid analgesic prescription claims for non-cancer chronic opioid users in an Illinois Medicaid managed care program in 2015-2016.

MAIN MEASURES: Per prescriber rates of singleprescriber high-daily-dose prescriptions or multiprescriber discoordination.

KEY RESULTS: For 2280 beneficiaries, 36,798 opioid prescription claims were submitted by 3532 prescribers. Compared to $3 \%$ of prescriptions (involving $6 \%$ of prescribers and $7 \%$ of beneficiaries) that directed $\geq 90$ $\mathrm{MME} /$ day, discoordination accounted for a greater share of high-risk exposures-13\% of prescriptions (involving $23 \%$ of prescribers and $24 \%$ of beneficiaries). The following specialties were at highest risk of discoordinated prescribing compared to internal medicine: dental (incident rate ratio (95\% confidence interval) $5.9(4.6,7.5))$, emergency medicine $(4.7(3.8,5.8))$, and surgical subspecialties (4.2 (3.0, 5.8)). Social network analysis identified 2 small interconnected prescriber communities of high-volume pain management specialists, and 3 sparsely connected groups of predominantly low-volume primary care or emergency medicine clinicians. Using multivariate models, we found that the sparsely connected sociometric positions were a risk factor for high-risk exposures.

\footnotetext{
Prior presentations Abstract presented at the Council of State and Territorial Epidemiologists (CSTE) annual meeting, June 2018

Electronic supplementary material The online version of this article (https://doi.org/10.1007/s11606-019-05114-3) contains supplementary material, which is available to authorized users.
}

Received September 20, 2018

Revised January 22, 2019

Accepted May 17, 2019

Published online August 16, 2019
CONCLUSION: Low-volume prescribers in the social network's periphery were at greater risk of intended or discoordinated prescribing than interconnected highvolume prescribers. Interventions addressing discoordination among low-volume opioid prescribers in non-integrated practices should be a priority. Demands for enhanced functionality and integration of Prescription Drug Monitoring Programs or referrals to specialized multidisciplinary pain management centers are potential policy implications.

KEY WORDS: opioid analgesic prescribing; social network analysis; care discoordination; epidemiology; harm reduction; Medicaid.

J Gen Intern Med 34(11):2443-50

DOI: $10.1007 / \mathrm{s} 11606-019-05114-3$

(c) Society of General Internal Medicine 2019

\section{INTRODUCTION}

The 2017 surveillance report from the Centers for Disease Control and Prevention documented the ongoing rise in overdose fatalities from opioid analgesics. ${ }^{1}$ This upward trend is remarkable considering that the rate of high-dose opioid analgesic prescriptions, defined as those directing $\geq 90$ morphine milligram equivalents (MME) per day, has been declining. ${ }^{1,2}$ A potential explanation of these discordant trends is that patients may be exposed to high-daily-dose opioids through the accumulation of prescriptions from multiple prescribers, each of whom may be guideline-adherent in terms of limiting the intended daily dosage.

Previous analyses of opioid prescription claims identified evidence of "pill mills" and "doctor shopping" in data. The California Workers' Compensation program (1993-2009) and Oregon Medicaid (2013) each revealed a disproportionate concentration of opioids prescribed by a small minority of clinicians for a few high-risk patients, indicative of the "pill mill" phenomenon. ${ }^{3,4}$ Such findings are tantalizing in that relatively quick fixes focused on a small number of culpable prescribers can be imagined. In contrast, analyses of an all-payor pharmacy claims database (2015) and Medicare Part D claims (2013) revealed that low-volume prescribers accounted for a significant 
number of high-risk exposures, and that "pill mills" may be regional phenomena or recently in decline. ${ }^{5,6}$ These findings suggest that discoordination among a network of prescribers contribute to risky cumulative daily opioid doses. The conditions associated with high-daily-dose or discoordinated prescribers are not well understood. ${ }^{7,8}$

To shed light on prescribers' behavior, we evaluated their risk of contributing to high-daily-dose or discoordinated opioid prescribing in the Chicago region using social network analysis. We used prescription claims data from CountyCare, an Illinois Medicaid Managed Care Community Network that had approximately 170,000 active beneficiaries in 2015-2016. We conducted our analysis guided by the following research questions: (1) What is the prevalence of high-daily-dose or discoordinated opioid analgesic prescribing? (2) What are the individual and social network characteristics of prescribers at risk for high-daily-dose or discoordinated opioid analgesic prescribing?

\section{METHODS}

\section{Data}

Prescription claims data for CountyCare beneficiaries in 2015-2016 contained the prescribed medication National Drug Code, dose quantity and date dispensed, prescriber, and duration of prescribed use. The National Drug Code was present for $99.99 \%$ of prescription claims. We included National Drug Codes corresponding to all oral opioids listed in the MME conversion table published by the Centers for Disease Control and Prevention. ${ }^{9}$ We excluded methadone and buprenorphine due to their more common use for the treatment of opioid use disorder rather than chronic pain disorder. We identified all beneficiaries with at least 90 consecutive days of prescribed opioid claims allowing no more than 14 continuous gap days (i.e., between the final date of intended receipt to the initiation date of the subsequent prescription). We excluded beneficiaries with cancer diagnosis codes. Prescribers were recognized using the National Provider Identifier (NPI) and we excluded NPI classified as organizations, or attributed to sub-specialists in oncology or palliative medicine. To facilitate reporting, we grouped nurse practitioners with physician assistants, pain specialists with anesthesiologists, physiatry with sports medicine specialists, maxillofacial surgeons with dentists, orthopedic with neurological surgery specialists, colorectal with general surgeons, and all other surgical specialists (podiatrists, urologists, otolaryngologists, ophthalmologists, dermatologists, plastic, vascular, thoracic, and hand surgeons) with one another.

\section{Network Construction and Visualization}

Numerous measures have been developed to determine each individual's role within a social network. For example, centrality measures the importance of individuals (nodes) within a population (network). ${ }^{10}$ Centrality measures may be based on the number of connections (ties) from each node to other nodes. In our network connecting prescribers and beneficiaries, for example, prescribers' centrality indicated their experience vis a vis the number of chronic users to whom opioids were prescribed, whereas beneficiaries' centrality indicated the potential for discoordination among multiple prescribers. Because the prescriber-beneficiary social network using our data is comprised of a highly connected small group of central nodes surrounded by nodes with decreasing centrality toward the periphery, we used the $k$-core method to label each node's sociometric position in the network. $K$-core layers a cored network like ours into nested levels involving nodes connected to at least $k$ other nodes. ${ }^{11} K$-shell is the part of the network contained in the rind of a $k$-core level that are sequentially removed to expose the next higher number of connections in the $(k+1)$-core until only the most connected inner core nodes remain. ${ }^{11,12}$

First, we created a bimodal matrix listing each prescriber and beneficiary on separate axes and indicated a relationship if $\geq 1$ opioid prescription was transacted in dyads. A $k$-shell level for each prescriber node in the network was assigned using UCINET6 (Analytic Technologies, Lexington KY). To visually represent the relationship between $k$-shell/core and the intensity of patient sharing, we created a separate matrix tabulating the number of shared patients between each pair of prescribers. Using R software, version 3.3.0 (R Core Team, R Foundation for Statistical Computing), we displayed this matrix as a heat map locating areas for discoordination potential in our network of prescribers. The heat map display was hierarchically sorted along the $x$ - and $y$-axes by $k$-shell/core (quantitatively strongest connections displayed in the top left section of the graph) and number of opioid prescription claims submitted (Fig. 1). We excluded prescriber observations with $<3$ shared patients to minimize uninformative empty spaces in the figure. We also constructed a traditional prescriber-level sociogram using Gephi 0.9.2 (The Gephi Consortium). We used the Fruchterman-Reingold and Yifan $\mathrm{Hu}$ algorithms to generate online Appendix A. ${ }^{13}$

\section{High-Daily-Dose and Discoordinated Opioid Analgesic Prescribing}

After calculating the daily MME for each opioid analgesic prescription, we tabulated the prevalence of prescriptions, prescribers, and beneficiaries associated with a $\geq 90 \mathrm{MME} /$ day exposure for at least 1 day. We defined two archetypal prescription-level events and two prescriber-level events by which the $90 \mathrm{MME} /$ day threshold could be exceeded. The prescription-level events were "single prescription high dose" (event A) involving individual prescriptions that directed patients to take $\geq 90 \mathrm{MME} /$ day, and "multiple overlapping prescription high dose" (event B), involving overlapping prescriptions days by one or more prescribers that additively exposed patients to $\geq 90 \mathrm{MME} / \mathrm{day}$. Prescriber-level events 


\section{Number of Shared \\ Beneficiaries}

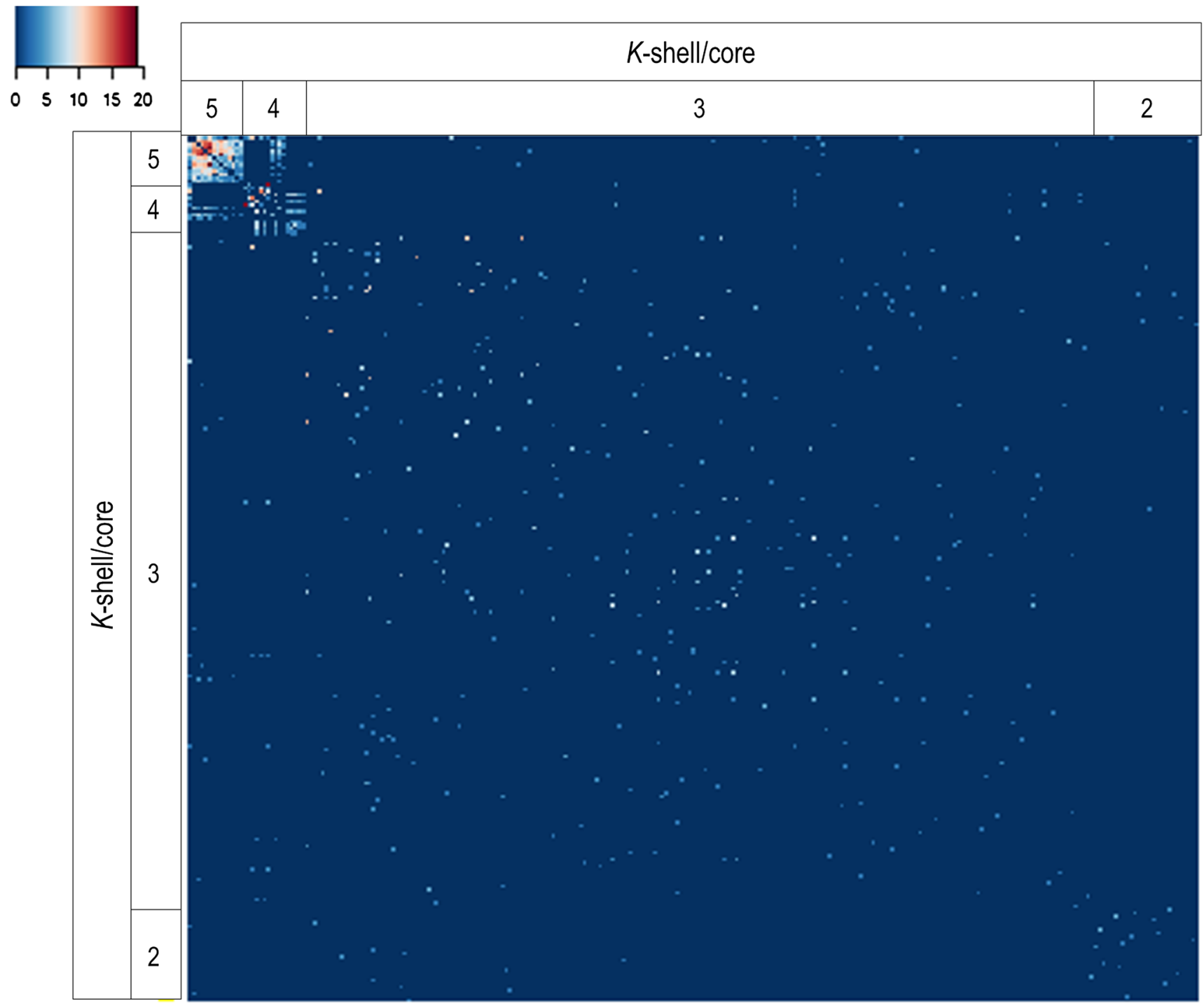

Fig. 1 Heat map displaying the number of shared beneficiaries between pairs of prescribers sharing at least 3 beneficiaries, sorted hierarchically along both axes by $k$-shell/core and the number of opioid prescription claims from top to bottom and left to right.

were our primary outcomes measure and used "prescribing episodes" or consecutive days of calculated uniform average daily MME exposure. All prescribing episodes were directed by a single prescriber; when $>1$ prescriptions overlapped by dates (e.g., separate prescriptions for long- and short-acting opioids, or for serial prescriptions filled early to avoid running out), we calculated the average daily MME through the duration of the overlapping prescriptions. We defined "single prescribing episode high dose" (event C) as a prescribing episode that exceeded $\geq 90 \mathrm{MME} /$ day. Event $\mathrm{C}$ was designed to detect high-daily-dose orders with greater expected sensitivity than event A by accounting for a prescriber's intended daily opioid exposure for a patient through multiple overlapping prescriptions. Finally, we defined "multiple prescriber discoordinator" (event D) as the prescriber of a prescribing episode that started while $\geq 90 \mathrm{MME}$ from an earlier prescribing episode by another prescriber remained. Event D was designed to identify discoordination more specifically than event B and is a situation that, theoretically, could be avoided through enhanced functionality of a Prescription Drug Monitoring Program database. We provided illustrative cases of these definitions in Fig. 2.

\section{Network Analysis}

We ran multivariate negative binomial regression with robust estimator of variance to assess the association between sociometric position (i.e., $k$-shell/core) and the number of high-risk exposures (separately for events C or D) using total number of opioid prescriptions for each prescriber as the offset variable ${ }^{14}$; we included $k$-shell/core and prescriber specialty as prescriber attributes. ${ }^{15}$ We used Stata14 (StataCorp, College Station, TX) 
Event A: Single Prescription High-Daily Dose Individual prescriptions directing patients to $\geq 90 \mathrm{MME} /$ day were counted. In the scenario, only Prescriber\#1's prescription met this criteria. The prevalence of Event $A$ in this scenario was 1 prescription involving 1 prescriber and 1 patient.
Event B: Multiple Prescription Overlapping High-Daily Dose

Multiple prescriptions by one or more prescribers additively directing patients to $\geq 90$ $\mathrm{MME} /$ day were counted. The 3 prescriptions by Prescribers \#1 and\#2 additively directed patients to $220 \mathrm{MME} /$ day during days1-5. Prescriptions by Prescribers \#2 and \#3 additively directed patients to $140 \mathrm{MME}$ day 6 and $100 \mathrm{MME}$ on day 7. The prevalence of Event $\mathrm{B}$ in this scenario was 4 prescriptions involving 3 prescribers and 1 patient.

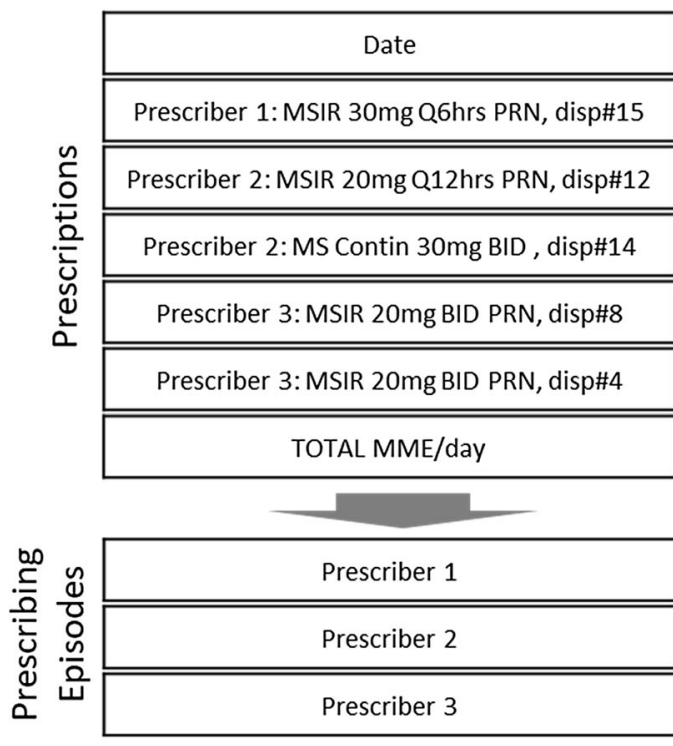

Event C: Single Prescribing Episode High-Daily Dose Prescribing episodes are consecutive days of uniform average daily MME exposures directed by a single prescriber.

Prescribing episodes directing patients to $\geq 90 \mathrm{MME} /$ day were counted. In the scenario, prescribing episodes by, both, Prescribers \#1 and \#2 met this criteria. The prevalence of Event $C$ in this scenario was 2 prescribing episodes involving 2 prescribers and 1 patient.

\begin{tabular}{|c|c|c|c|c|c|c|c|c|c|}
\hline 1 & 2 & 3 & 4 & 5 & 6 & 7 & 8 & 9 & 10 \\
\hline ' & 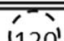 & 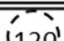 & 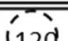 & "' & $<$ & & & & \\
\hline 120 & 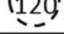 & 120 & $1 \underbrace{120}$ & 1120 & & & & & \\
\hline 40 & 40 & 40 & 40 & 40 & 40 & 6 & & & \\
\hline & & & & & & & & & \\
\hline 60 & 60 & 60 & 60 & 60 & 60 & 60 & & & \\
\hline & & & & & 40 & 40 & 40 & $40<$ & \\
\hline & & & & & & & & 40 & 40 \\
\hline 'ي & 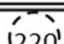 & 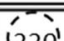 & "न्্ & "न्र & "' & & 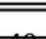 & & \\
\hline (2), & 1220 & 1220 & $1 \leq 29$ & 1220 & 1140 & 100 & 40 & 80 & 40 \\
\hline
\end{tabular}

Fig. 2 Illustrative scenario with definition of the four methods for calculating exposures of $\geq 90$ morphine milligram equivalents (MME)/day for a hypothetical patient.

for all statistical analyses. The study protocol was approved by the local Institutional Review Board.

\section{RESULTS}

Of all adult beneficiaries with any CountyCare prescription claim, $2280(1 \%)$ beneficiaries without a cancer diagnosis satisfied the criterion for chronic opioid analgesic use. Opioid prescription claims for these beneficiaries were submitted by 3532 individual prescribers, on average, one-and-a-half prescribers per beneficiary. The most common prescriber specialties were internal medicine, nurse practitioner or physician assistant, emergency medicine, and family medicine (Table 1$)$. About one-third ( $n=$ $1285,36.3 \%$ ) of prescribers submitted at least 4 opioid prescription claims. A substantial number of prescribers ( $n=1419,40.2 \%)$ were independent clinicians without a recorded organizational affiliation.

The prevalence of prescriptions, prescribers, and beneficiaries involved in $\geq 90 \mathrm{MME} /$ day exposures is shown in Table 2. Of 36,798 opioid prescription claims in our analysis, $3.1 \%$ directed beneficiaries to take $\geq 90 \mathrm{MME} /$ day (event $\mathrm{A}$ ), but about 4 times as many prescriptions, prescribers, and
Table 1 Characteristics of Opioid Analgesic Prescribers for Noncancer Beneficiaries with Chronic Opioid Use Enrolled in CountyCare 2015-2016

\begin{tabular}{|c|c|}
\hline Distinct prescribers, $N$ & 3532 \\
\hline \multicolumn{2}{|l|}{ Clinical specialty, $n(\%)$} \\
\hline Internal medicine and specialties & $804(22.8)$ \\
\hline Nurse practitioner/physician assistant & $540(15.3)$ \\
\hline Emergency medicine & $537(15.2)$ \\
\hline Family medicine & $476(13.5)$ \\
\hline No specialty & $278(7.9)$ \\
\hline Dental specialties & $211(6.0)$ \\
\hline Orthopedic/neurological surgery & $170(4.8)$ \\
\hline Anesthesia/pain & $116(3.3)$ \\
\hline Other surgical specialties & $113(3.2)$ \\
\hline General/colorectal surgery & $78(2.2)$ \\
\hline Physiatry/sports medicine & $66(1.9)$ \\
\hline Obstetrics/gynecology & $42(1.2)$ \\
\hline Other & $37(1.1)$ \\
\hline Psychiatry & $32(0.9)$ \\
\hline Neurology & $22(0.6)$ \\
\hline Pediatrics & $10(0.3)$ \\
\hline \multicolumn{2}{|c|}{ Number of opioid prescriptions per prescriber quintiles, $n(\%)$} \\
\hline $1: 1-1$ & $1410(39.9)$ \\
\hline $2: 2-2$ & $533(15.1)$ \\
\hline $3: 3-3$ & $304(8.6)$ \\
\hline $4: 4-10$ & $623(17.6)$ \\
\hline $5: 11-587$ & $662(18.7)$ \\
\hline \multicolumn{2}{|l|}{$K$-shell, $n(\%)$} \\
\hline 1 & $2240(63.4)$ \\
\hline 2 & $837(23.7)$ \\
\hline 3 & $425(12.0)$ \\
\hline 4 & $16(0.5)$ \\
\hline 5-core & $14(0.4)$ \\
\hline
\end{tabular}


Table 2 Prevalence of Opioid Analgesic Prescriptions, Prescribers, and Beneficiaries Associated with Daily Opioid Exposures of $\geq 90$ mg Morphine Equivalents (MME) Per Day Among Non-cancer Beneficiaries with Chronic Opioid Use Enrolled in CountyCare 2015-2016

\begin{tabular}{|c|c|c|c|c|c|c|}
\hline \multirow{6}{*}{$\begin{array}{l}\text { Opioid prescriptions, } \mathrm{N}_{1} \\
\text { Event A: single prescription high daily dose, } n\left(\% \text { of } \mathrm{N}_{1}\right) \\
\text { Event B: multiple prescription overlapping high daily dose, } n\left(\% \text { of } \mathrm{N}_{1}\right) \\
\text { Opioid prescribing episodes } \dagger, \mathrm{N}_{2} \\
\text { Event C: single prescribing episode high daily dose, } n\left(\% \text { of } \mathrm{N}_{2}\right) \\
\text { Event D: multiple prescribing episode discoordinator, } n\left(\% \text { of } \mathrm{N}_{2}\right)\end{array}$} & \multicolumn{2}{|c|}{ Prescriptions } & \multicolumn{2}{|c|}{ Prescribers } & \multicolumn{2}{|c|}{ Beneficiaries } \\
\hline & 36,798 & & 3532 & & 2280 & \\
\hline & & $1143(3.1)$ & & $221(6.3)$ & & $167(7.3)$ \\
\hline & & $4690(12.8)^{*}$ & & $803(22.7)^{*}$ & & $553(24.3)$ \\
\hline & 26,566 & & 3532 & & 2280 & \\
\hline & & $2361(8.9)+$ & & $1316(37.3) \ddagger$ & & 949 (41.6) \\
\hline
\end{tabular}

beneficiaries were involved in additive daily exposures of $\geq 90$ $\mathrm{MME} /$ day due to overlapping prescription days (event B). The total MME dispensed by individual prescriptions directing patients to take $\geq 90 \mathrm{MME} /$ day (event A) was 4.5 million MME, whereas the total MME dispensed by all overlapping prescriptions (event B) was 16 million MME, 3.5 million MME of which were dispensed exclusively on days when at least 2 prescriptions overlapped. The prevalence of singleprescriber high-daily-doses was similar when counting prescriptions (event A) or prescribing episodes (event C). Discoordinators (event D) were identified in $8.9 \%$ of prescribing episodes involving $37.3 \%$ of prescribers and $41.5 \%$ of beneficiaries.

The median amount remaining from a previous prescribing episode when these discoordinator episodes were dispensed was 297 (interquartile range 160 to 659) MME.

\section{Prescribing Patterns by $k$-Shell/Core}

In the social network, 4 shells around one core were assigned, yielding a total of 5 sociometric levels (Table 1). The majority of prescribers were in the least connected shell (1-shell) and a much smaller number were in the tightly connected 4-shell and 5-core (online Appendix A). The majority of prescribers in the 4-shell and 5-core were anesthesia-pain specialists affiliated with 2 separate hospital-based chronic pain clinics. The large majority of prescribers in the $3 k$-shells in the periphery of the provider network were primary care clinicians or emergency medicine specialists, and a plurality were prescribers not affiliated with a practice organization.

As shown in Table 3, prescribing patterns differed across the $k$-shells/core; despite the smaller aggregate number of prescriptions by clinicians in the inner compared to outer $k$-shells, each inner $k$-shell/core clinician submitted a greater number of prescription claims that dispensed more MME and for many more high-risk beneficiaries than clinicians in the outer $k$-shells. However, despite the greater intensity of prescribing, the proportion of prescriptions contributing to high daily exposures trended smaller for the inner compared to the outer $k$ shells.

\section{Risk of Single-Provider High-Daily-Dose Pre- scribing Episodes}

Prescribers in 2- with 3-shell, and 4-shell with 5-core were grouped to facilitate reporting. The models on the left side of Table 4 indicated prescribers in the outermost $k$-shell associated with a greater adjusted risk of high-daily-dose prescribing, whereas the core was associated with smaller risk. Dentists and pediatricians did not issue a prescribing episode that surpassed the $90 \mathrm{MME} /$ day threshold. Compared to internal medicine specialists, emergency medicine specialists were less likely to issue prescribing episodes exceeding $90 \mathrm{MME} /$ day (IRR $(95 \%$ CI) $0.17(0.07,0.43))$. In contrast, prescribers were at greater risk of high-daily-dose prescribing episodes among physiatry or sports medicine specialists $(2.35(1.41,3.91))$ and anesthesiology or pain specialists $(2.47(1.46,4.19))$.

\section{Risk of Discoordinator Prescribing Episodes}

As delineated in models on the right side of Table 4, a stepwise reduction in the bivariate risk of issuing a discoordinating prescription claim was seen for the inner compared to outermost $k$-shell. This relationship became statistically not significant when adjusted for prescriber specialty, due partially to model instability from high degree of correlation between the independent variables. Compared to internal medicine specialists, physicians with the highest risk of contributing to discoordinator prescribing episodes were dental specialists (IRR (95\% CI) $5.85(4.59,7.45))$, emergency medicine specialists $(4.65(3.75,5.77))$, other surgical specialists $(4.08$ $(2.95,5.63))$, orthopedic and neurosurgeons (3.54 (2.69, $4.67)$ ), and general surgeons $(3.03(2.04,4.49))$. Prescribers without a National Provider Index specialty taxonomy and non-physician prescribers were also associated with greater risk of a discoordinator prescribing episode.

The significance of the statistical findings above was further illustrated in Fig. 1. In the heat map representing the degree of patient sharing by pairs of prescribers, disproportionately intense patient sharing was evident among all of the 5-core prescribers compared to the outer shells where patient sharing was much more sporadic. This finding illustrated that the beneficiaries shared among the 5-core prescribers rarely received prescriptions from prescribers outside of the 5-core 
Table 3 Prescribing Patterns by $k$-shell/Core Among Prescribers of Opioid Analgesics for Non-cancer Beneficiaries with Chronic Opioid Use Enrolled in CountyCare 2015-2016

\begin{tabular}{|c|c|c|c|c|c|c|c|}
\hline $\begin{array}{l}\text { K- } \\
\text { shell }\end{array}$ & $\begin{array}{l}\text { Prescribers, } \\
n\end{array}$ & $\begin{array}{l}\text { Prescribing } \\
\text { episodes, } n\end{array}$ & $\begin{array}{l}\text { Prescribing } \\
\text { episode per } \\
\text { prescriber, } \\
\text { median (min, } \\
\text { IQR, max) }\end{array}$ & $\begin{array}{l}\text { Beneficiaries per } \\
\text { prescriber, } \\
\text { median (min, } \\
\text { IQR, max) }\end{array}$ & $\begin{array}{l}\text { Total MME } \\
\text { dispensed per } \\
\text { prescriber, median } \\
\text { (IQR) }\end{array}$ & $\begin{array}{l}\text { High daily } \\
\text { dose, } n \text { (\% } \\
\text { prescribing } \\
\text { episode) }\end{array}$ & $\begin{array}{l}\text { Discoordinator, } n \\
\text { (\% prescribing } \\
\text { episode) }\end{array}$ \\
\hline 1 & 2240 & 5758 & $1(1,1,2109)$ & $1(1,1,2,6)$ & $300(111,1200)$ & $279(5)$ & $679(12)$ \\
\hline 2 & 837 & 7236 & $4(2,2,12,290)$ & $2(2,2,3,19)$ & $1152(290,6000)$ & $237(3)$ & $624(9)$ \\
\hline 3 & 425 & 11,195 & $12(3,6,38,587)$ & $6(3,4,9,50)$ & $5475(1050,22,585)$ & $258(2)$ & $873(8)$ \\
\hline 4 & 16 & 971 & $\begin{array}{l}43(7,19.5 \\
67.5452)\end{array}$ & $16.5(4,9,27,45)$ & $\begin{array}{l}22,362(18,095 \\
56,217)\end{array}$ & $16(2)$ & $67(7)$ \\
\hline $\begin{array}{l}5- \\
\text { core }\end{array}$ & 14 & 1406 & $\begin{array}{l}110(49,66,154, \\
512)\end{array}$ & $39(20,31,51,55)$ & $\begin{array}{l}69,721(41,460 \\
90,240)\end{array}$ & $0(0)$ & $118(8)$ \\
\hline
\end{tabular}

Key: min, minimum; IQR, interquartile range; max, maximum

furthering our observation that they behaved not as "doctor shoppers" but as regular patients of a tightly interconnected network of pain management specialists. Despite patient sharing representing the potential for discoordination, the lower risk of a discoordinator prescribing episode suggested risk mitigation among tightly interconnected prescribers. Conversely, despite sparse patient sharing in the periphery, the relatively high discoordination risk among loosely connected prescribers indicated areas requiring remedy.

\section{DISCUSSION}

Using prescription claims data from a large Medicaid managed care plan, we found that high-risk exposures from discoordinated prescribing by multiple prescribers were more prevalent as intended high daily doses from a single prescriber. In our study population, discoordination was more prevalent by the number of prescriptions, prescribers, beneficiaries, and MME involved. Social network analysis uncovered that discoordinated prescribing was more prevalent in the less interconnected parts of the prescriber-beneficiary social network, and that there was a tightly connected core group of pain management specialists who prescribed the most MME, but with the least risk of exposing patients to high-risk dosages. Among clinician specialties, the risk of discoordinated prescribing was particularly elevated among dentists, emergency medicine specialists, and surgical specialists.

Existing interventions targeting clinicians to limit the MME they prescribe can be broadly categorized into those that target individual prescribers and those designed to aid coordination among prescribers around individual patients. ${ }^{16}$ Public health

Table 4 Incident Rate Ratios (IRR) and 95\% Confidence Intervals (95\% CI) for Prescriber Attributes Informing Risk of Exposing Beneficiaries to Opioid Analgesics at $\geq 90$ MME/Day Through High-Daily-Dose or Discoordinator Prescribing Episodes

\begin{tabular}{|c|c|c|c|c|}
\hline \multirow[t]{2}{*}{ Dependent variable } & \multicolumn{2}{|c|}{ Rate of high-daily-dose prescribing episodes } & \multicolumn{2}{|c|}{ Rate of discoordinator prescribing episodes } \\
\hline & $\begin{array}{l}\text { Bivariate IRR } \\
(95 \% \mathrm{CI})\end{array}$ & $\begin{array}{l}\text { Multivariate IRR } \\
(95 \% \text { CI })\end{array}$ & $\begin{array}{l}\text { Bivariate IRR } \\
(95 \% \text { CI })\end{array}$ & $\begin{array}{l}\text { Multivariate IRR } \\
\text { (95\% CI) }\end{array}$ \\
\hline \multicolumn{5}{|l|}{$K$-shell } \\
\hline 1 & $1.79(1.32,2.43)$ & $1.97(1.45,2.69)$ & $1.20(1.05,1.38)$ & $1.13(1.00,1.29)$ \\
\hline $\begin{array}{l}2 \\
3\end{array}$ & REF & $\mathrm{REF}$ & $\mathrm{REF}$ & $\mathrm{REF}$ \\
\hline $\begin{array}{l}4 \\
5 \text {-core }\end{array}$ & $1.02(0.29,3.64)$ & $0.39(0.16,0.96)$ & $0.61(0.47,0.79)$ & $0.96(0.70,1.31)$ \\
\hline \multicolumn{5}{|l|}{ Specialty } \\
\hline Internal medicine and specialties & REF & REF & REF & REF \\
\hline $\begin{array}{l}\text { Nurse practitioner/physician as- } \\
\text { sistant }\end{array}$ & $0.73(0.44,1.20)$ & $0.84(0.53,1.33)$ & $2.10(1.65,2.68)$ & $2.11(1.66,2.68)$ \\
\hline Emergency medicine & $0.15(0.05,0.39)$ & $0.17(0.07,0.43)$ & $4.68(3.77,5.82)$ & $4.65(3.75,5.77)$ \\
\hline Family medicine & $0.72(0.44,1.16)$ & $0.78(0.50,1.23)$ & $0.76(0.58,0.99)$ & $0.77(0.58,1.01)$ \\
\hline No specialty & $0.40(0.17,0.95)$ & $0.43(0.17,1.09)$ & $2.49(1.87,3.31)$ & $2.45(1.84,3.26)$ \\
\hline Dental specialties ${ }^{\mathrm{a}}$ & na & na & $5.97(4.697 .61)$ & $5.85(4.59,7.45)$ \\
\hline Orthopedic/neurological surgery & $0.80(0.38,1.70)$ & $0.95(0.51,1.77)$ & $3.58(2.71,4.72)$ & $3.54(2.69,4.67)$ \\
\hline Anesthesia/pain & $1.83(0.93,3.59)$ & $2.47(1.46,4.19)$ & $1.12(0.83,1.51)$ & $1.16(0.82,1.63)$ \\
\hline Other surgical specialties & $0.30(0.07,1.19)$ & $0.28(0.06,1.20)$ & $4.19(3.03,5.80)$ & $4.08(2.95,5.63)$ \\
\hline General/colorectal surgery & $0.63(0.17,2.34)$ & $0.69(0.17,2.68)$ & $3.11(2.08,4.64)$ & $3.03(2.04,4.49)$ \\
\hline Physiatry/sports medicine & $2.14(0.88,5.19)$ & $2.35(1.41,3.91)$ & $0.91(0.50,1.66)$ & $0.91(0.50,1.66)$ \\
\hline Obstetrics/gynecology & $0.40(0.04,4.23)$ & $0.32(0.03,3.93)$ & $2.07(0.86,4.99)$ & $1.93(0.80,4.64)$ \\
\hline Other & $0.30(0.04,2.33)$ & $0.33(0.05,2.15)$ & $1.59(0.83,3.07)$ & $1.57(0.82,3.01)$ \\
\hline Psychiatry & $0.55(0.10,3.02)$ & $0.47(0.13,1.73)$ & $1.31(0.52,3.32)$ & $1.25(0.49,3.16)$ \\
\hline Neurology & $1.16(0.23,5.82)$ & $1.17(0.31,4.36)$ & $1.38(0.41,4.67)$ & $1.28(0.38,4.34)$ \\
\hline Pediatrics ${ }^{\mathrm{a}}$ & na & na & $0.38(0.03,5.40)$ & $0.37(0.03,5.05)$ \\
\hline
\end{tabular}

${ }^{a}$ No prescribing episodes in these specialties directed $\geq 90$ MME/day 
detailing (the use of trained educators) and clinical practice guidelines are examples of strategies targeting individual prescribers. ${ }^{17}$ Our analysis suggests that prescribers who may benefit most from public health detailing are least exposed to high-risk patients and practice more often at the periphery of social networks, which admittedly make educational outreach efforts more complicated. The educational content for specialists in dentistry, emergency medicine, and surgical specialties along with primary care clinicians should also inform them about strategies to mitigate their recognized vulnerabilities to care fragmentation. ${ }^{18,}{ }^{19}$ Emergency medicine prescribers among them should be a primary target for interventions because they frequently provide opioid prescriptions. Prescription Drug Monitoring Programs, on the other hand, serve to directly aid coordination of care by allowing prescribers to see the MME prescribed by other clinicians. Existing evaluations of these strategies' effectiveness have yielded mixed results, ${ }^{20-23}$ but measuring the effect of legal mandates for prescribers to use Prescription Drug Monitoring Programs is an important future investigation, particularly in Illinois where legislation for mandatory use went into effect in $2018 .^{24}$ Technological improvements in the form of seamless access to the Prescription Drug Monitoring Program through electronic health record systems is another promising development. ${ }^{25}$ Finally, innovations to reduce discoordinated prescribing also can apply to pharmacists, who could play a role addressing discoordinated dispensing in parallel to discoordinated prescribing. ${ }^{26}$

Additionally, our analysis shed light on the potential advantages of integrated specialty practices. We found the tightly connected 5-core of our network was primarily comprised of clinicians staffing an interdisciplinary pain management center on the central campus of a public healthcare delivery system. Although these clinicians prescribed opioid analgesics to more high-risk patients by raw numbers than other clinicians, and their specialty was associated with greater risk of high-dailydose prescriptions, these clinicians behaved not as "pill mills" but managed to expose fewer patients to high daily doses and mitigated the rate of discoordination in spite of their greater vulnerability due to high volume. Their success illuminates the potential of practice integration reinforced by normalized practice behaviors, a shared electronic health record, and experience-based proficiency to protect against "doctor shopping." ${ }^{27,}{ }^{28}$ Success may also be related to their multidisciplinary approach to the treatment of chronic pain involving intensive behavioral therapists, integrative medicine specialists in acupuncture, and interventional anesthesia physicians. Addressing the referral capacity of non-opioid-centric interdisciplinary pain management centers like this one may be important in some locales and as part of a national solution.

The strengths of our study were in the use of detailed prescribing data across a large cohort of beneficiaries and the novel application of social network analysis to better understand discoordinated opioid prescribing. However, we do need to acknowledge several limitations. First, $\geq 90 \mathrm{MME} /$ day exposure from one or more prescriptions do not necessarily indicate deviant prescribing behavior or inappropriate use by patients. Second, prescribed and actual uses of opioid analgesics may differ, but our primary focus was on prescriber behavior. Third, the quality of prescriber attribute data linked to the National Provider Index has not been validated, though the data has been used in many studies. Fourth, the presence of multicollinearity demands cautious interpretation of regression coefficients. Each of our claims is supported by our bivariate analysis where the multivariate models are unreliable. ${ }^{29}$ Finally, we believe insights from our analysis may appropriately inform opioid analgesic prescribing behavior in other settings; however, geographic and population variability threaten direct generalizability of our findings. Specifically, Chicago has experienced a disproportionately larger exposure to illicit opioids compared to prescription opioids than the rest of the USA. ${ }^{30}$ The interactions between illicit opioid exposure and demand for opioid analgesics are complex and incompletely understood. $^{31}$

A clinician's decision to prescribe opioids for a patient with chronic non-cancer pain involves myriad considerations that cannot be viewed in isolation. ${ }^{32}$ We evaluated features of the social network comprising prescribers and patients that inform the risk of prescriptions contributing to high daily exposures. The useful insights gleaned from our analysis is that much of the prescribing behavior that results in high daily exposures for patients is related to discoordinated care, which will not be solved through adherence to practice guidelines alone. Also, tightly connected groups of prescribers were better at avoiding highdose and discoordinated prescribing. Public health programs and policy development should be cognizant of these challenges and potential solutions.

Corresponding Author: Keiki Hinami, MD, MS; Department of Medicine Cook County Health, Chicago, IL, USA (e-mail: khinami@cookcountyhhs.org).

Additional Contributions Momin M. Malik, $\mathrm{PhD}$, provided advice on data visualization.

Funding/Support The Illinois Department of Healthcare and Family Services approved the research use of CountyCare data. Cook County Health supported this project.

\section{Compliance with Ethical Standards:}

Conflict of Interest: The authors declare that they do not have a conflict of interest.

\section{REFERENCES}

1. 2018 Annual Surveillance Report of Drug-Related Risks and Outcomes United States. Centers for Disease Control and Prevention, US Department of Health and Human Services. Published August 31, 2018. 
https://www.cdc.gov/drugoverdose/pdf/pubs/2018-cdc-drug-surveillance-report.pdf. Accessed 1 May 2019

2. Nuckols TK, Anderson L, Popescu I, et al. Opioid prescribing: a systematic review and critical appraisal of guidelines for chronic pain. Ann Intern Med 2014;160:38-47.

3. Prescribing patterns of schedule II opioids in California Workers' Compensation. 2011. http://www.cwci.org/document.php?file=1438. pdf. Accessed 1 May 2019

4. Kim H, Hartung DM, Jacob RL, McCarty D, McConnell KJ. The Concentration of Opioid Prescriptions by Providers and Among Patients in the Oregon Medicaid Program. Psychiatr Serv 2016;67:397-404.

5. Chang HY, Murimi IB, Jones CM, Alexander GC. Relationship between high-risk patients receiving prescription opioids and high-volume opioid prescribers. Addiction 2017.

6. Chen JH, Humphreys $\mathbf{K}$, Shah NH, Lembke A. Distribution of Opioids by Different Types of Medicare Prescribers. JAMA Intern Med 2016;176:259-61.

7. Rolheiser LA, Cordes J, Subramanian Sv. Opioid Prescribing Rates by Congressional Districts, United States, 2016. Am J Public Health 2018;108:1214-9.

8. Dijkstra A, Hak E, Janssen F. A systematic review of the application of spatial analysis in pharmacoepidemiologic research. Ann Epidemiol 2013;23:504-14.

9. Opioid Overdose Data Resources. 2017. https://www.cdc.gov/ drugoverdose/resources/data.html. Accessed 1 May 2019

10. Scott J. SOCIAL NETWORK ANALYSIS. THIRD EDITION. ed. Los Angeles: SAGE; 2013.

11. Borgatti SP, Everett MG, Johnson JC. Analyzing social networks. Los Angeles: SAGE; 2013.

12. Seidman SB. Network structure and minimum degree. Soc Networks 1983;5:269-87.

13. Bastian M, Heymann S, Jacomy M. Gephi: an open source software for exploring and manipulating networks. International AAAI Conference on Weblogs and Social Media 2009

14. Wagner B, Riggs P, Mikulich-Gilbertson S. The importance of distribution-choice in modeling substance use data: a comparison of negative binomial, beta binomial, and zero-inflated distributions. Am J Drug Alcohol Abuse 2015;41:489-97.

15. Rice E, Barman-Adhikari A, Milburn NG, Monro W. Position-specific HIV risk in a large network of homeless youths. Am J Public Health 2012;102:141-7.

16. Barth KS, Guille C, McCauley J, Brady KT. Targeting practitioners: A review of guidelines, training, and policy in pain management. Drug Alcohol Depend 2017;173 Suppl 1:S22-S30.

17. Kattan JA, Tuazon E, Paone D, et al. Public Health Detailing-A Successful Strategy to Promote Judicious Opioid Analgesic Prescribing. Am J Public Health 2016;106:1430-8.

18. Barnett ML, Olenski AR, Jena AB. Opioid-Prescribing Patterns of Emergency Physicians and Risk of Long-Term Use. N Engl J Med 2017;376:663-73.
19. McCauley JL, Hyer JM, Ramakrishnan VR, et al. Dental opioid prescribing and multiple opioid prescriptions among dental patients: Administrative data from the South Carolina prescription drug monitoring program. J Am Dent Assoc 2016;147:537-44.

20. Haegerich TM, Paulozzi LJ, Manns BJ, Jones CM. What we know, and don't know, about the impact of state policy and systems-level interventions on prescription drug overdose. Drug Alcohol Depend 2014;145:3447.

21. Lin HC, Wang Z, Boyd C, Simoni-Wastila L, Buu A. Associations between statewide prescription drug monitoring program (PDMP) requirement and physician patterns of prescribing opioid analgesic for patients with non-cancer chronic pain. Addict Behav 2018 348-54.

22. Bao Y, Pan Y, Taylor A, et al. Prescription Drug Monitoring Programs Are Associated With Sustained Reductions In Opioid Prescribing By Physicians. Health Aff (Millwood) 2016;35:1045-51.

23. Deyo RA, Hallvik SE, Hildebran C, et al. Association of Prescription Drug Monitoring Program Use With Opioid Prescribing and Health Outcomes: A Comparison of Program Users and Nonusers. J Pain 2018; 19:166-77.

24. Haffajee RL, Jena AB, Weiner SG. Mandatory use of prescription drug monitoring programs. JAMA 2015;313:891-2.

25. Bolton D, Sabel J, Baumgartner C, Kushner T, Karras BT. Using Health Information Exchange to Improve Use of Prescription Monitoring Data. Online J Public Health Inform 2017;9:e145.

26. Yang Z, Wilsey B, Bohm M, et al. Defining risk of prescription opioid overdose: pharmacy shopping and overlapping prescriptions among longterm opioid users in medicaid. J Pain 2015;16:445-53.

27. Kitahata MM, Koepsell TD, Deyo RA, Maxwell CL, Dodge WT, Wagner EH. Physicians' experience with the acquired immunodeficiency syndrome as a factor in patients' survival. N Engl J Med 1996;334:701-6.

28. McDonald DC, Carlson KE. The ecology of prescription opioid abuse in the USA: geographic variation in patients' use of multiple prescribers (“doctor shopping"). Pharmacoepidemiol Drug Saf 2014;23:1258-67.

29. Vatcheva KP, Lee M, McCormick JB, Rahbar MH. Multicollinearity in Regression Analyses Conducted in Epidemiologic Studies. Epidemiology (Sunnyvale) 2016;6.

30. Chicago Department of Public Health. Epidemiology report: Increase in overdose deaths involving opioids - Chicago, 2015-20162017 October.

31. Compton WM, Jones CM, Baldwin GT. Relationship between Nonmedical Prescription-Opioid Use and Heroin Use. N Engl J Med 2016;374:154-63.

32. Toye F, Seers $\mathbf{K}$, Tierney $\mathbf{S}$, Barker $\mathbf{K L}$. A qualitative evidence synthesis to explore healthcare professionals' experience of prescribing opioids to adults with chronic non-malignant pain. BMC Fam Pract 2017;18:94.

Publisher's Note: Springer Nature remains neutral with regard to jurisdictional claims in published maps and institutional affiliations. 